a relationship which has been checked experimentally by Miller. ${ }^{12}$ In those cases where the above interfacial tension inequality is violated, so that lens formation is impossible, two limiting cases arise. When $\gamma_{\mathrm{I} \mathrm{III}}>\gamma_{\mathrm{I} \text { II }}$ $+\gamma_{\text {II III }}$ the middle phase spreads out as a film, while

${ }^{12}$ N. F. Miller, J. Phys. Chem. 45, 1025 (1941). for $\gamma_{\text {II III }}>\gamma_{\text {I II }}+\gamma_{\text {I III }}$ the middle phase forms a globule which floats by buoyancy. ${ }^{13}$

Thus, it is seen that even simple applications of the Neumann formula provide a thermodynamic foundation for various phenomena encountered in surface chemistry.

${ }^{13}$ W. M. Coghill and C. O. Anderson, U. S. Bureau of Mines, Technical Paper 262 (1923).

\title{
Hindered Rotation in Molecules with Relatively High Potential Barriers*
}

\author{
KarL T. Hecht and David M. Dennison \\ Harrison M. Randall Laboratory of Physics, University of Michigan, Ann Arbor, Michigan
}

(Received March 19, 1956)

\begin{abstract}
The theory of hindered rotation has been applied to the type of asymmetric molecule in which the hindering barrier is high enough so that the hindered rotation splittings of the energy levels are small compared with the rotational energies but yet large enough to be observable in the microwave spectrum. The specific type of molecule considered consists of a rigid asymmetric component which may undergo a hindered rotation about the symmetry axis of a rigid symmetric component where the symmetric component is in addition assumed to have threefold symmetry and the asymmetric component at least a plane of symmetry containing the symmetry axis of the symmetric component. An example might be the acetaldehyde molecule, $\mathrm{CH}_{3} \mathrm{CHO}$.

In principle, the theory developed by Burkhard and Dennison can be used directly but in practice the method is difficult to apply to such a molecule since the matrix elements of the Hamiltonian
\end{abstract}

\section{INTRODUCTION}

$I^{\mathrm{N}}$ $\mathrm{N}$ a series of papers from this laboratory ${ }^{1-3}$ the theory of hindered rotation has been developed and applied to the methyl alcohol molecule, $\mathrm{CH}_{3} \mathrm{OH}$. The details of the methyl alcohol spectrum are influenced very markedly by the fact that the molecule is a nearly symmetric rotator in which the potential barrier hindering the internal rotation is a relatively low one. The probability of the tunneling process associated with the hindering barrier is such that the hindered rotation splittings of the energy levels are of the order of magnitude of the rigid rotator energies themselves and much greater than the asymmetry splittings of these levels.

In the present paper the theory of hindered rotation will be applied to a type of molecule falling into a somewhat different category, namely an asymmetric molecule with a relatively high potential barrier in which the hindered rotation splittings are small compared with the rotational energies but yet large enough to be ob-

* The preparation of this manuscript was supported by Contract Nonr-1224(15) between the Office of Naval Research and the Engineering Research Institute of The University of Michigan.

1 J. S. Koehler and D. M. Dennison, Phys. Rev. 57, 1006 (1940).

2 D. G. Burkhard and D. M. Dennison, Phys. Rev. 84, 408 (1951).

${ }^{3}$ E. V. Ivash and D. M. Dennison, J. Chem. Phys. 21, 1804 (1953). used previously do not degenerate naturally or easily to those for the rigid asymmetric rotator in the infinite barrier limit. In the present treatment a transformation is made on the Hamiltonian whereby this complication is avoided and the resulting calculations are greatly simplified.

It is found that the spectrum is essentially that of the rigid rotator with the important exception that all the strong lines are split into two components. For the low $J$ transitions specific formulas have been derived for these splittings which are relatively simple functions of the barrier height, the principal moments of inertia, and two additional parameters involving the molecular dimensions and the masses. The barrier height can thus be deduced from the observed splittings without the use of the somewhat cumbersome machinery needed in the general case.

servable in the microwave spectrum. For the type of molecule to be considered the rotational lines will be given in zeroth order through the asymmetric rigid rotator energy levels alone. The chief effect of the hindered rotation is to produce a splitting of these lines, and it will be shown that the height of the hindering barrier in this simple case can be determined quite easily from rather few experimental data and without the use of the somewhat cumbersome machinery needed in the general case.

Specifically, the molecules to be considered are assumed to consist of a rigid asymmetric component which may undergo a hindered rotation about the symmetry axis of a rigid symmetric component. In addition the symmetric component is assumed to have three-fold symmetry and the asymmetric component at least a plane of symmetry containing the symmetry axis of the symmetric component. A model of such a molecule is shown in Fig. 1. ${ }^{4}$

\footnotetext{
${ }^{4}$ A generalization to molecules consisting of a completely asymmetric component linked to a symmetric component will be discussed briefly in an appendix. The generalization to molecules in which the symmetric component has $k$-fold symmetry, with $k>3$, is very straightforward. However, if $k=2$, the molecule will in general consist of two asymmetric components, and although the methods used can, in principle, be extended to this case also, the theory becomes very complicated due to the complete asymmetry of both components.
} 


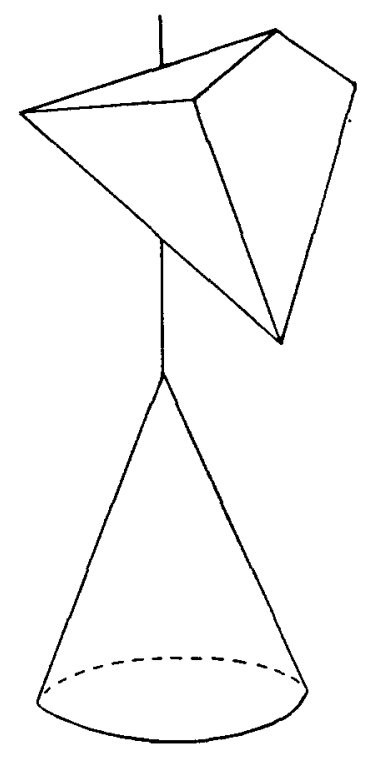

Fig. 1. Model of the type of molecule discussed.

There exist many molecules (for example, the substituted ethanes such as $\left.\mathrm{CH}_{3} \mathrm{CH}_{2} \mathrm{~F}\right)$ which have the required symmetry properties, but of these only a relatively small proportion will have a barrier height of just such a magnitude that the tunneling frequencies are small compared with the rotational frequencies and yet large enough to be observable. This circumstance has its origin in the extreme sensitivity of the tunneling effect to the barrier height. As will be shown the acetaldehyde molecule, $\mathrm{CH}_{3} \mathrm{CHO}$, may be a molecule satisfying all these requirements.

In principle, the theory developed in the paper by Burkhard and Dennison ${ }^{2}$ can be used directly, but in practice the method is very difficult to apply to the high barrier case since the matrix elements of the Hamiltonian in this formulation do not degenerate naturally or easily to those for the rigid asymmetric rotator in the case of an infinitely high barrier. The difficulty arises from the fact that there is an internal angular momentum associated with the hindered rotation with respect to a framework of rotating axes defined by the symmetry axis of the hindering potential which from the point of view of the molecular forces is a natural choice. For this reason the earlier form of the Hamiltonian contains cross products between the momentum associated with the hindered rotation and the components of total angular momentum. These crossproduct terms lead to matrix elements off-diagonal in $n$, the limiting vibrational quantum number of the torsional motion, which in the high barrier limit cannot be neglected, even in the zeroth order of the rotational energies. The degeneration to the correct zeroth order rigid rotator comes about through these $n$ to $n^{\prime}$ connections so that the solution of even the zeroth-order problem involves a complicated diagonalization process.

In the present treatment a transformation is made on the Hamiltonian whereby these complications are avoided. The matrix elements of the transformed Hamiltonian may be expressed in a form such that, in the high barrier limit, they become those of the correct rigid asymmetric rotator plus those of a simple hindered rotator plus a small number of perturbation terms. A simple hindered rotator is defined as one whose wave equation is

$$
\frac{-\hbar^{2}}{2 \mu} \frac{d^{2}}{d x^{2}} M(x)+V(x) M(x)=E_{\text {internal }} M(x),
$$

where $x$ is the internal rotation angle giving the relative orientation of the two components of the molecule, $\mu$ is some reduced moment of inertia depending on the two components of the molecule, and $M(x)$ is the internal rotation part of the wave function. For the specific type of molecule to be considered the hindering potential has the form $V(x)=H / 2(1-\cos 3 x)$. A sinusoidal form will be used for the hindering potential here as in the earlier treatments. Deviations from this form can only introduce minor corrections in the type of molecule under consideration.

Except for the transformation of the Hamiltonian and certain approximations valid in the high barrier case, the method of solution is that given in the paper by Burkhard and Dennison. It consists of taking matrix elements of the Hamiltonian using as a basis the wave functions which are exact solutions of the symmetric hindered rotator part of the Hamiltonian. The diagonalization of these matrix elements then yields the energy levels and the correct wave functions of the asymmetric hindered rotator. The basis wave functions are specified by the usual symmetric rigid rotator quantum numbers, $J, K$, and $M$, and the two hindered rotation quantum numbers, $n$ and $\tau$, where $\tau$ can have the values 1,2 , or 3 and is the quantum number describing the threefold splitting of the energy levels due to the tunneling effect associated with the three-fold hindering barrier.

The structure of the energy levels for the various types of rotators has been discussed before ${ }^{3}$ and is shown in Fig. 2 to illustrate the structure of the levels and the transitions for the type of molecule of present interest. The energy levels of a symmetric rigid rotator (SRR), shown on the left, are split into three as a finite three-fold hindering barrier is introduced. Both the energy levels and the transitions for the symmetric hindered rotator (SHR) occur in groups of three. (The particular transitions shown here will occur only if the molecule has a component of dipole moment perpendicular to the symmetry axis.) These levels are all doubly degenerate, but the introduction of the asymmetry causes a splitting of only one of these levels. For a molecule such as methyl alcohol with a relatively low barrier (lb) and a very small asymmetry (sa) both the levels of the asymmetric hindered rotator (AHR) and the allowed transitions giving rise to the strong lines occur in groups of four. If the barrier height is imagined to go to infinity these four levels must go over to the 
two asymmetric rigid rotator (ARR) levels. However, if the barrier is not too high each of these levels is split into two components, one a doubly degenerate one $(E)$, the other a nondegenerate one $\left(A_{1}\right.$ or $\left.A_{2}\right)$. For relatively high barriers (hb) and large enough asymmetries (la) the hindered rotation splittings will be much less than the asymmetry splittings, and the spectrum will essentially be that of an asymmetric rigid rotator with the important exception that all the strong lines of the spectrum, like the energy levels, are split into two components where these are of comparable intensity. There will, in addition, be certain allowed transitions giving rise to single lines which would be forbidden for the rigid rotator and which can therefore be expected to be very much weaker.

In this limiting case of high barriers specific formulas can be given, for the low $J$ values at least, for the splittings of the energy levels, and from these formulas the barrier height can be deduced directly if the principal moments of inertia of the molecule are known from an analysis of the gross features of the spectrum. In addition there may be a shift of the center of gravity of each pair of hindered rotation levels from the position of the limiting rigid rotator level. Relatively little use can be made of these shifts of the levels, however, since they may be affected considerably by all the other vibration-rotation perturbations of the problem.

\section{TRANSFORMATION OF THE HAMILTONIAN}

The transformation to the new Hamiltonian will be made in a form where the physical meaning of the transformation is readily apparent. The kinetic energy for the type of molecule of present interest is ${ }^{2}$

$$
2 T=A \omega_{x}{ }^{2}+B \omega_{y}{ }^{2}+C \omega_{z}{ }^{2}-2 D \omega_{y} \omega_{z}-2 C_{2} \omega_{z} \dot{x}+C_{2} \dot{x}^{2},
$$

where $C_{2}$ is the moment of inertia of the symmetric component about its symmetry axis, and where the moments of inertia of the entire molecule, $A, B, C$, and the product of inertia, $D$, are defined with respect to a system of orthogonal axes fixed in the asymmetric component of the molecule with origin at the center of mass of the whole molecule. The $z$ axis is parallel to the symmetry axis of the hindering potential, and the $y$ axis lies in the plane of symmetry of the asymmetric component of the molecule. The vector $\omega$ denotes the angular velocity of the asymmetric component of the molecule and can be expressed in terms of the Euler angles, $\theta, \psi$, and $\phi_{1}$ describing the orientation in space of the asymmetric component. The angular velocity of the symmetric component of the molecule has been eliminated by the introduction of the internal rotation angle, $x=\phi_{1}-\phi_{2}$, where $\phi_{1}$ and $\phi_{2}$ are the azimuth angles of the two parts of the molecule about the symmetry axis of the hindering potential.

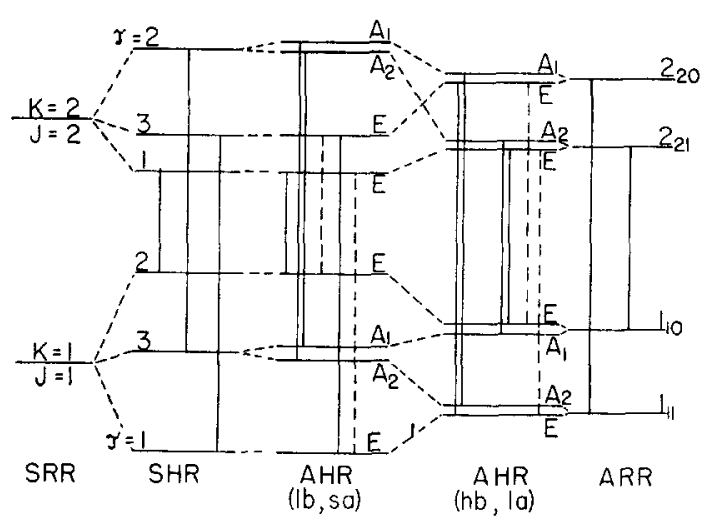

FIG. 2. Structure of the energy levels for the various types of rotators. SRR - symmetric rigid rotator; SHR-symmetric hindered rotator; AHR-asymmetric hindered rotator; (lb, sa)low barrier, small asymmetry, (hb, la) - high barrier, large asymmetry; ARR - asymmetric rigid rotator. The dashed lines indicate allowed transitions which can be expected to be very weak.

The classical Hamiltonian becomes

$$
\begin{gathered}
H=\frac{P_{x}^{2}}{2 A}+\frac{P_{y}^{2} C_{1}}{2\left(B C_{1}-D^{2}\right)}+\frac{P_{z}^{2} B}{2\left(B C_{1}-D^{2}\right)} \\
+\frac{P_{y} P_{z} D}{\left(B C_{1}-D^{2}\right)}+\frac{p_{x} P_{y} D}{\left(B C_{1}-D^{2}\right)}+\frac{p_{x} P_{z} B}{\left(B C_{1}-D^{2}\right)} \\
+\frac{p_{x}^{2}\left(B C-D^{2}\right)}{2 C_{2}\left(B C_{1}-D^{2}\right)}+V(x),
\end{gathered}
$$

where

$$
p_{x}=\frac{\partial T}{\partial \dot{x}}, \quad P_{x}=\frac{\partial T}{\partial \omega_{x}}, \cdots
$$

and where $P_{x}, P_{y}$, and $P_{z}$ are given in terms of the canonical variables $p_{\theta}, p_{\psi}, p_{\phi 1}, \theta, \psi$, and $\phi_{1}$ by the usual relations. It can be shown that $P_{x}, P_{y}$, and $P_{z}$ are the components of the total angular momentum of the molecule expressed with respect to the system of axes fixed in the asymmetric portion of the molecule. However, the total angular momentum receives contributions from the internal rotation. The canonical variable, $p_{x}$, conjugate to the internal rotation angle, $x$, is a function of more than just the relative motion of the two parts of the molecule. It does not, for example, give the angular momentum of the symmetric component relative to the asymmetric component.

Also, in this form the Hamiltonian is clearly not that of the correct rigid rotator plus that of a simple hindered rotator with only small rotation-hindered rotation perturbation terms since it is the moment of inertia $C_{1}$ rather than $C$ which occurs in the terms involving $P_{y}$ and $P_{z}$. The difficulty arises from the fact that there is an internal angular momentum associated with the relative rotation of the two groups of the molecule when viewed from a framework of axes rotating with the 
asymmetric portion. (An observer located on the asymmetric component of the molecule would say that the symmetric component has an angular momentum relative to his coordinate system.) The difficulty is overcome if the relative rotation of the two components of the molecule is viewed from a system of axes which rotate in space in such a way that no internal angular momentum is associated with the mutual rotation of the two groups. This system of axes which describes the rotation of the whole molecular framework in space will be called the "molecule-fixed" system of axes although its orientation relative to either of the two rigid components of the molecule depends on the internal rotation angle $x$. In the limit in which classically $x \rightarrow 0=$ constant, it will indeed become a system of axes fixed in the whole molecule. However, for this type of asymmetric molecule it will not coincide with a system of axes defined by the symmetry axis of the hindering potential, and it need not coincide with the principal axes of inertia of the rigid molecule.

In the ordinary rotating and vibrating molecule any coordinate system fixed in the limiting rigid molecule can serve as a proper rotating framework for the description of the vibrational motions since the condition of zero internal angular momentum can always be satisfied by an appropriate choice of the internal degrees of freedom. In hindered rotators, however, the physics of the situation dictates the choice of the internal degrees of freedom, and since these initially may not be free of internal angular momentum with respect to coordinate systems which seem most natural from the point of view of the molecular forces, a proper "molecule-fixed" system of axes must first be found if the rotation-hindered rotation interaction terms in the Hamiltonian are to appear as small perturbation terms. This can be accomplished by a transformation on the Hamiltonian which eliminates the cross-product terms of the form $p_{x} P_{i}$.

The situation is simplest for symmetric hindered rotators or for molecules such as nitromethane, $\mathrm{CH}_{3} \mathrm{NO}_{2},{ }^{5,6}$ in which the symmetry axis of the hindering potential coincides with one of the principal axes of inertia of the whole molecule. For such a molecule the moleculefixed system of axes is defined by the symmetry axis of the hindering potential and the new azimuth angle $\phi^{\prime}=\left(C_{1} / C\right) \phi_{1}+\left(C_{2} / C\right) \phi_{2}$. When viewed from this system of axes the internal angular momentum due to the rotation of one component of the molecule is canceled by the equal and opposite internal angular momentum of the other component of the molecule.

In terms of the angular momentum components, the transformation to the "molecule-fixed" system for a

${ }^{5}$ Tannenbaum, Johnson, Myers, and Gwinn, J. Chem. Phys. 22, 949 (1954).

${ }^{6}$ Wilson, Lin, and Lide, J. Chem. Phys. 23, 136 (1955). symmetric hindered rotator is

$$
\begin{gathered}
p_{x}=p_{x}{ }^{\prime}-\left(\frac{C_{2}}{C}\right) P_{z}^{\prime} \\
\left.\left[\begin{array}{l}
P_{x} \\
P_{y} \\
P_{z}
\end{array}\right]=\left(\begin{array}{ccc}
\cos \left(\frac{C_{2}}{C} x\right) & \sin \left(\frac{C_{2}}{C} x\right) & 0 \\
-\sin \left(\frac{C_{2}}{C} x\right) & \cos \left(\frac{C_{2}}{C} x\right) & 0 \\
0 & 0 & 1
\end{array}\right]\right)\left(\begin{array}{l}
P_{x}^{\prime} \\
P_{y}^{\prime} \\
P_{z}^{\prime}
\end{array}\right] .
\end{gathered}
$$

In this case the transformation is described much more simply in terms of the Euler angles

$$
\begin{gathered}
\theta^{\prime}=\theta, \quad \psi^{\prime}=\psi, \quad x^{\prime}=x \\
\varphi^{\prime}=\varphi_{1}-\left(\frac{C_{2}}{C}\right) x \quad\left[=\frac{C_{1}}{C} \varphi_{1}+\frac{C_{2}}{C} \varphi_{2}\right] .
\end{gathered}
$$

This was the transformation proposed by Nielsen ${ }^{7}$ which leads to the symmetric hindered rotator Hamiltonian used by Koehler and Dennison.

The transformation to the molecule-fixed system for the asymmetric hindered rotator is somewhat more complicated but can be accomplished by a rotation of axes in the $y-z$ plane followed by a new choice of azimuth angle about the new $z$ axis. The rotation in the $y-z$ plane is chosen in such a way as to eliminate the cross term between $p_{x}$ and $P_{y}$ in the Hamiltonian, Eq. (3). The new $p_{x} P_{z}$ term can then be eliminated by a subsequent proper choice for the new azimuth angle. The transformation to the molecule-fixed system is now

$$
p_{x}=p_{x}^{\prime}-\left(\frac{C_{2}}{C}\right)^{*} P_{z}^{\prime}
$$

$\left[\begin{array}{l}P_{x} \\ P_{y} \\ P_{z}\end{array}\right]=\left[\begin{array}{ccc}1 & 0 & 0 \\ 0 & \frac{B}{\left(B^{2}+D^{2}\right)^{\frac{1}{2}}} & \frac{D}{\left(B^{2}+D^{2}\right)^{\frac{1}{2}}} \\ 0 \frac{-D}{\left(B^{2}+D^{2}\right)^{\frac{1}{2}}} & \frac{B}{\left(B^{2}+D^{2}\right)^{\frac{1}{2}}}\end{array}\right)$

$$
\times\left[\begin{array}{ccc}
\cos \left(\frac{C_{2}}{C}\right)^{*} x & \sin \left(\frac{C_{2}}{C}\right)^{*} x & 0 \\
-\sin \left(\frac{C_{2}}{C}\right)^{*} x & \cos \left(\frac{C_{2}}{C}\right)^{*} x & 0 \\
0 & 0 & 1
\end{array}\right]\left(\begin{array}{c}
P_{x}^{\prime} \\
P_{y}^{\prime} \\
P_{z}^{\prime}
\end{array}\right)
$$

\footnotetext{
${ }^{7}$ H. H. Nielsen, Phys. Rev. 40, 445 (1932).
} 
where

$$
\left(\frac{C_{2}}{C}\right)^{*}=\frac{C_{2}\left(B^{2}+D^{2}\right)^{\frac{1}{2}}}{\left(B C-D^{2}\right)}
$$

The $P_{i}{ }^{\prime}$ are the new components of the total angular momentum vector. Now, however, it can be shown that the total angular momentum of the asymmetric molecule arises solely from the rotation in space of the whole molecular framework as defined by the molecule-fixed system of axes; and the new momentum variable, $p_{x}{ }^{\prime}$, associated with the hindered rotation is a function only of the relative motion of the two parts of the molecule. [Classically, $\left.p_{x}{ }^{\prime}=C_{2}\left(B C_{1}-D^{2}\right) \dot{x} /\left(B C-D^{2}\right).\right]$

The new momenta must and do satisfy the usual
Poisson bracket relations

and cyclically,

$$
\left(P_{x}^{\prime}, P_{y}^{\prime}\right)=P_{z}^{\prime}
$$

and

$$
\left(P_{i}^{\prime}, p_{x}^{\prime}\right)=0, \quad\left(P_{i}^{\prime}, x^{\prime}\right)=0 .
$$

The angular momentum components $P_{x}{ }^{\prime}, P_{y}^{\prime}$, and $P_{z}{ }^{\prime}$ are now given in terms of the new canonically conjugate variables, $p_{\theta}{ }^{\prime}, p_{\psi}{ }^{\prime}, p_{\phi}{ }^{\prime}$, and $\theta^{\prime}, \psi^{\prime}, \phi^{\prime}$, by the usual relations, where $\theta^{\prime}, \psi^{\prime}$, and $\phi^{\prime}$ are the Euler angles defining the orientation in space of the new molecule-fixed system of axes. The functional relationship between these angles and the old angles $\theta, \psi, \phi_{1}$, and $x$ is quite complicated and has the following form:

$$
\begin{aligned}
& \cos \theta^{\prime}=\frac{B}{\left(B^{2}+D^{2}\right)^{\frac{1}{2}}} \cos \theta+\frac{D}{\left(B^{2}+D^{2}\right)^{\frac{1}{2}}} \sin \theta \cos \varphi_{1}, \\
& \tan \psi^{\prime}=\frac{\tan \psi\left(\sin \theta-\frac{D}{B} \cos \theta \cos \varphi_{1}\right)-\frac{D}{B} \sin \varphi_{1}}{\left(\sin \theta-\frac{D}{B} \cos \theta \cos \varphi_{1}\right)+\frac{D}{B} \sin \varphi_{1} \tan \psi} \\
& \tan \left[\varphi^{\prime}+\left(\frac{C_{2}}{C}\right)^{*} x\right]=\frac{\sin \varphi_{1}\left(B^{2}+D^{2}\right)^{\frac{1}{2}}}{B \cos \varphi_{1}-D \cot \theta} \\
& x^{\prime}=x \text {. } \\
& +\frac{1}{4}\left(\frac{1}{A}-\frac{B}{B^{2}+D^{2}}\right)\left[\left(P_{x}^{\prime 2}-P_{y}^{\prime 2}\right) \cos 2\left(\frac{C_{2}}{C}\right)^{*} x\right. \\
& \left.+\left(P_{x}^{\prime} P_{y}^{\prime}+P_{y}^{\prime} P_{x}^{\prime}\right) \sin 2\left(\frac{C_{2}}{C}\right)^{*} x\right] \\
& \text { II } \\
& +\frac{1}{2} \frac{D}{\left(B^{2}+D^{2}\right)}\left[\left(P_{u}^{\prime} P_{z}^{\prime}+P_{z}^{\prime} P_{y}^{\prime}\right) \cos \left(\frac{C_{2}}{C}\right)^{*} x\right. \\
& \left.-\left(P_{x}^{\prime} P_{z}^{\prime}+P_{z}^{\prime} P_{x}^{\prime}\right) \sin \left(\frac{C_{2}}{C}\right)^{*} x\right] \text {. }
\end{aligned}
$$

The new variables $p_{\theta}{ }^{\prime}, p_{\psi}{ }^{\prime}, p_{\phi}{ }^{\prime}, p_{x}{ }^{\prime}, \theta^{\prime}, \psi^{\prime}, \phi^{\prime}$, and $x^{\prime}$ satisfy the Poisson bracket relations required for canonical variables. The transformation is therefore a valid contact transformation.

This transformation leads directly to the new form of the classical Hamiltonian, but in order to solve the quantum mechanical problem the Hamiltonian must be expressed in correct symmetrized quantum mechanical form. The angular momentum components, $P_{i}{ }^{\prime}$, and the momentum $p_{x}^{\prime}$ must be replaced by the usual operators which satisfy the commutation relations corresponding to Eqs. (7). In the representation in which $P^{\prime 2}$ and $P_{z}{ }^{\prime}$ are diagonal the matrix element of the $P_{i}^{\prime}$ have the customary form in terms of the rigid rotator quantum numbers $J$ and $K$. They are independent of the hindered rotator quantum numbers $n$ and $\tau$ since the $P_{i}^{\prime}$ commute with $p_{x}{ }^{\prime}$ and $x^{\prime}$.

The quantum mechanical form of the new Hamiltonian is

$$
I\left\{\begin{aligned}
H= & \frac{1}{4}\left(\frac{1}{A}+\frac{B}{B^{2}+D^{2}}\right)\left(P_{x}^{\prime 2}+P_{y}^{\prime 2}\right) \\
& +\frac{1}{2}\left(\frac{B+C}{B C-D^{2}}-\frac{B}{B^{2}+D^{2}}\right) P_{z}^{\prime 2} \\
& +\frac{1}{2} \frac{\left(B C-D^{2}\right)}{C_{2}\left(B C_{1}-D^{2}\right)} p_{x}{ }^{2}+V(x)
\end{aligned}\right.
$$

It will be noticed that there are now no terms of the form $p_{x}{ }^{\prime} P_{i}{ }^{\prime}$, and in the high barrier limit where classically $x \rightarrow 0=$ constant, the Hamiltonian degenerates directly to that of the correct rigid rotator plus that of a simple hindered rotator. At this point a transformation to principal axes of inertia could still be made. However, the hindered rotator matrix elements of the Hamiltonian will be much simpler in the present form, Eq. (9). The transformation to the principal axis form of the limiting rigid molecule will be made at a later stage in the calculation. Since the internal rotation angle, $x$, is not affected by the transformation, the form of the hindering potential is unchanged.

Part I of the Hamiltonian again has the form of the symmetric hindered rotator discussed in the paper by 
Koehler and Dennison, provided the reciprocal moment of inertia $1 / A$ is replaced by $1 / 2\left[1 / A+B /\left(B^{2}+D^{2}\right)\right]$ and $1 / C$ is replaced by $\left[(B+C) /\left(B C-D^{2}\right)-B /\left(B^{2}+D^{2}\right)\right]$. In addition, the reduced moment of inertia of the simple hindered rotator which was $C_{1} C_{2} / C$ for symmetric molecules must now be replaced by $C_{2}\left(B C_{1}-D^{2}\right) /$ $\left(B C-D^{2}\right)$. The interaction between the hindered rotation and the rotation of the whole molecule in space will come about mainly through the boundary conditions, but since part II of the Hamiltonian, which arises from the asymmetry of the molecule, still contains the internal rotation angle $x$, part of the rotation-hindered rotation interaction comes about through these terms in the Hamiltonian itself. However, this interaction may be shown to be a very mild one as the barrier height becomes large.

The method of solution consists of taking matrix elements of the Hamiltonian using as a basis the exact solutions of part I which have the form

$$
\Psi=\frac{1}{2 \pi} \Theta\left(\theta^{\prime}\right) e^{i M \psi^{\prime}} e^{i K \varphi^{\prime}}\left[e^{i \sigma x} P_{K r n}(x)\right],
$$

where $P_{K \tau n}(x)$ is a periodic function of $x$, and where the value of $\sigma$ is determined by the boundary conditions. ${ }^{1}$ The wave function must be invariant when $\phi_{1} \rightarrow \phi_{1}+2 n_{1} \pi$ and $\phi_{2} \rightarrow \phi_{2}+2 n_{2} \pi$ where $n_{1}$ and $n_{2}$ are any integers, since the physical situation is unaltered by this transformation. In terms of the new variables, $\phi^{\prime}$ and $x$, this transformation becomes

$$
\varphi^{\prime} \rightarrow \varphi^{\prime}-\left(\frac{C_{2}}{C}\right)^{*}\left(n_{1}-n_{2}\right) 2 \pi, \quad x \rightarrow x+\left(n_{1}-n_{2}\right) 2 \pi .
$$

The wave function will be invariant to this transformation if

$$
-K\left(\frac{C_{2}}{C}\right)^{*}\left(n_{1}-n_{2}\right)+\sigma\left(n_{1}-n_{2}\right)=n,
$$

where $n$ is an integer. This equation will hold if $K$ is an integer, and if

$$
\sigma=-K\left[1-\frac{C_{2}\left(B^{2}+D^{2}\right)^{\frac{1}{2}}}{\left(B C-D^{2}\right)}\right]=-K\left(\frac{C_{1}}{C}\right)^{*}
$$

(The choice of $\sigma$ depends on the choice of the integer $n$ which is arbitrary. Here it is chosen so that $\sigma$ goes over to the Koehler-Dennison value when $D=0$.)

\section{MATRIX ELEMENTS OF THE HAMILTONIAN}

Using these wave functions as a basis the matrix elements of the Hamiltonian become

$$
\begin{aligned}
& H_{J K r n}^{J K \tau n}=\frac{\hbar^{2}}{4}\left(\frac{1}{A}+\frac{B}{B^{2}+D^{2}}\right)\left(J^{2}+J-K^{2}\right)+\frac{\hbar^{2}}{2}\left(\frac{B+C}{B C-D^{2}}-\frac{B}{B^{2}+D^{2}}\right) K^{2}+E_{K r n}{ }^{\text {int }}, \\
& H_{J K \tau n} J(K+1) r^{\prime} n^{\prime}=\frac{\hbar^{2}}{4} \frac{D}{B^{2}+D^{2}}(2 K+1)[(J-K)(J+K+1)]^{\frac{1}{2}} \int_{0}^{2 \pi} P_{K \tau n^{*}}(x) e^{-i x} P_{(K+1) r^{\prime} n^{\prime}}(x) d x, \\
& H_{J K r n} J(K+2) \tau^{\prime \prime \prime} n^{\prime}=-\frac{h^{2}}{8}\left(\frac{1}{A}-\frac{B}{B^{2}+D^{2}}\right)[(J-K)(J-K-1)(J+K+1)(J+K+2)]^{\frac{1}{2}} \\
& \times \int_{0}^{2 \pi} P_{K \tau n}{ }^{*}(x) e^{-2 i x} P_{(K+2) \tau^{\prime \prime} n^{\prime}}(x) d x .
\end{aligned}
$$

The allowed $\tau$ connections are exactly the same as those given in the paper by Burkhard and Dennison. ${ }^{2}$ The energy determinant again factors into three subdeterminants where the $\tau$ value associated with a particular $K$ value is uniquely defined for each subdeterminant. Two of the determinants have identical roots giving rise to levels of species $E$, while the third gives rise to nondegenerate levels of species $A$.

In these matrix elements no high barrier approximation has been made as yet. ${ }^{8}$ However, as the barrier becomes high the integrals involving the internal rotation angle $x$ (with $n^{\prime}=n$ ) become equal to unity plus

\footnotetext{
8 The diagonalization of the matrix elements is easier in this new form even if the barrier to hindered rotation is a relatively low one. The $n \rightarrow n^{\prime}$ connections $\left(n^{\prime} \neq n\right)$ besides being simpler in form, now give only very small contributions to the energies for any state lying appreciably below the top of the barrier, and even for states lying above the top of the barrier the $n \rightarrow n^{\prime}$ connections will be smaller than those occurring in the earlier formulation.
}

small negative perturbation terms; and, except for these perturbation terms and even smaller perturbation terms with $n^{\prime} \neq n$, the matrix elements are those of an asymmetric rigid rotator to which is added the internal energy $\left(E_{K r n}{ }^{\text {int }}\right)$ of a simple hindered rotator. This internal energy must be computed with the new value for $\sigma$ and the modified value for the reduced moment of inertia, but the method of solution is unchanged. ${ }^{1}$ The rigid rotator matrix elements are not expressed with respect to principal axes of inertia. They could have been so expressed if the contact transformation (6) had been followed by a rotation to principal axes. In that case however the energy determinant would not have been split into three subdeterminants, and the complicated connections between the three determinants would have been considerably more difficult to handle than the $K \rightarrow K \pm 1$ connections which arise 
because the rigid rotator part of the Hamiltonian is not expressed with respect to principal axes of inertia.

In any case, the rigid rotator part of the Hamiltonian can be diagonalized for each individual $J$ value. For the low $J$ 's this can be done for general values of the moments of inertia. The procedure will be illustrated in detail for $J=1$. If $S$ is the unitary matrix which diagonalizes the rigid rotator part of the matrix elements, $H$, in the form (12), then the new matrix elements, $H^{\prime}$, given by $H^{\prime}=S^{-1} H S$, will be diagonal in the infinite barrier limit. For $J=1$

$$
S=S_{1} S_{2}=\left(\begin{array}{ccc}
\frac{1+\alpha}{2} & \frac{\beta}{\sqrt{2}} & \frac{1-\alpha}{2} \\
-\frac{\beta}{\sqrt{2}} & \alpha & \frac{\beta}{\sqrt{2}} \\
\frac{1-\alpha}{2} & -\frac{\beta}{\sqrt{2}} & \frac{1+\alpha}{2}
\end{array}\right)\left(\begin{array}{ccc}
-\frac{1}{\sqrt{2}} & 0 & \frac{1}{\sqrt{2}} \\
0 & 1 & 0 \\
\frac{1}{\sqrt{2}} & 0 & \frac{1}{\sqrt{2}}
\end{array}\right)
$$

where

$$
\begin{aligned}
\left\{\begin{array}{l}
\alpha^{2} \\
\beta^{2}
\end{array}\right\} & =\frac{1}{2} \pm \frac{1}{2} \frac{(B-C)\left(B^{2}-D^{2}\right)+4 B D^{2}}{\left(B^{2}+D^{2}\right)\left[(B-C)^{2}+4 D^{2}\right]^{\frac{1}{2}}} \\
\alpha \beta & =\frac{D\left(B C-D^{2}\right)}{\left(B^{2}+D^{2}\right)\left[(B-C)^{2}+4 D^{2}\right]^{\frac{1}{2}}}
\end{aligned}
$$

and where $\alpha$ and $\beta$ are the direction cosines of the $z$-principal axis with respect to the $z^{\prime}$ and $y^{\prime}$ moleculefixed axes defined by transformation (6).

The matrix elements of the transformed Hamiltonian, $H^{\prime}$, will again be factored into groups of three $(2 J+1)$ by $(2 J+1)$ submatrices corresponding to determinants 1 , 2, and 3. These are shown explicitly for $J=1$ in Table I. Determinant 3, giving the nondegenerate energy levels, splits into two further subdeterminants corresponding to the symmetries $A_{1}$ and $A_{2}$ to be discussed in a later section. In Table I, $a, b$, and $c$ are the reciprocals of the principal moments of inertia (times $\hbar^{2} / 2$ ) and are related to the moments and product $A$, $B, C$, and $D$ by the following expressions

$$
\begin{aligned}
& a=\frac{\hbar^{2}}{2 A_{\text {princ }}}=\frac{\hbar^{2}}{2 A}, \\
& b=\frac{\hbar^{2}}{2 B_{\text {princ }}}=\frac{\hbar^{2}}{2} \frac{(B+C)-\left[(B-C)^{2}+4 D^{2}\right]^{\frac{1}{2}}}{2\left(B C-D^{2}\right)} \\
& c=\frac{\hbar^{2}}{2 C_{\text {princ }}}=\frac{\hbar^{2}}{2} \frac{(B+C)+\left[(B-C)^{2}+4 D^{2}\right]^{\frac{1}{2}}}{2\left(B C-D^{2}\right)} .
\end{aligned}
$$

Again, no high barrier approximation has been made as yet, but the matrix elements are now in a form suitable for a perturbation treatment in the high barrier case. The quantities

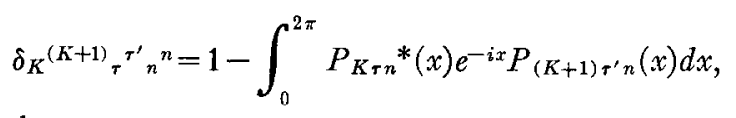

and

$$
\begin{aligned}
\delta_{K^{(K+2)} \tau^{\tau^{\prime \prime}}{ }_{n}{ }^{n}} & \\
& =1-\int_{0}^{2 \pi} P_{K \tau n} *(x) e^{-2 i x} P_{(K+2) \tau^{\prime \prime} n}(x) d x
\end{aligned}
$$

go to zero as the barrier height goes to infinity as do also the differences $\left(E_{K r n}-E_{K \tau^{\prime} n}\right)$. The diagonal elements now give the energy levels up to and including first-order perturbation terms. Higher-order perturbation terms can be brought in by the usual techniques. An ordinary perturbation treatment will be valid only if the hindered rotation splittings are small compared to the asymmetry splittings of the limiting rigid rotator. However, matrix elements in this form can be used directly also to determine the hindered rotation energies in the special case when some of the limiting rigid rotator levels occur in closely spaced pairs, for example in the case of a nearly symmetric molecule. In that case the separation of two rigid rotator levels may be of the same order of magnitude as the hindered rotation splittings, and the off-diagonal matrix elements connecting these two limiting rigid rotator levels cannot be neglected. A special perturbation treatment will be needed for these levels similar to that for two vibrational levels in Fermi resonance, for example.

Formulas for the energies are therefore rather complicated in the case when the asymmetry splitting is of the same order of magnitude as the hindered rotation splitting, and the matrix elements in the form of Table $I$ are consequently most useful when the hindered rotation splittings of the levels are much smaller than the asymmetry splittings.

Matrix elements similar to those given in Table I for $J=1$ can be derived for any $J$ value. The $S$ matrix which diagonalizes the rigid rotator part of the Hamiltonian for a particular $J$ value can always be chosen to have the form $S=S_{1} S_{2}$, where $S_{1}$ transforms the rigid rotator part of the matrix elements from the form (12), containing zeroth order $K \rightarrow K \pm 1$ connections, to the usual form in which the rigid rotator Hamiltonian is expressed with respect to principal axes, and in which the zeroth-order off-diagonal matrix elements involve $K \rightarrow K \pm 2$ connections only. $S_{2}$, on the other hand, is the matrix which diagonalizes the asymmetric rigid rotator Hamiltonian when expressed with respect to principal axes. The $S_{2}$ matrices are therefore well known. ${ }^{9}$ They depend only upon the principal moments of inertia although they are quite complicated for the higher $J$ values.

The matrices $S_{1}$ will be functions only of $\alpha$ and $\beta$,

\footnotetext{
${ }^{9}$ King, Hainer, and Cross, J. Chem. Phys. 11, 27 (1943).
} 
TABLE I. Matrix elements of the Hamiltonian for $J=1$, diagonalized in zeroth order.

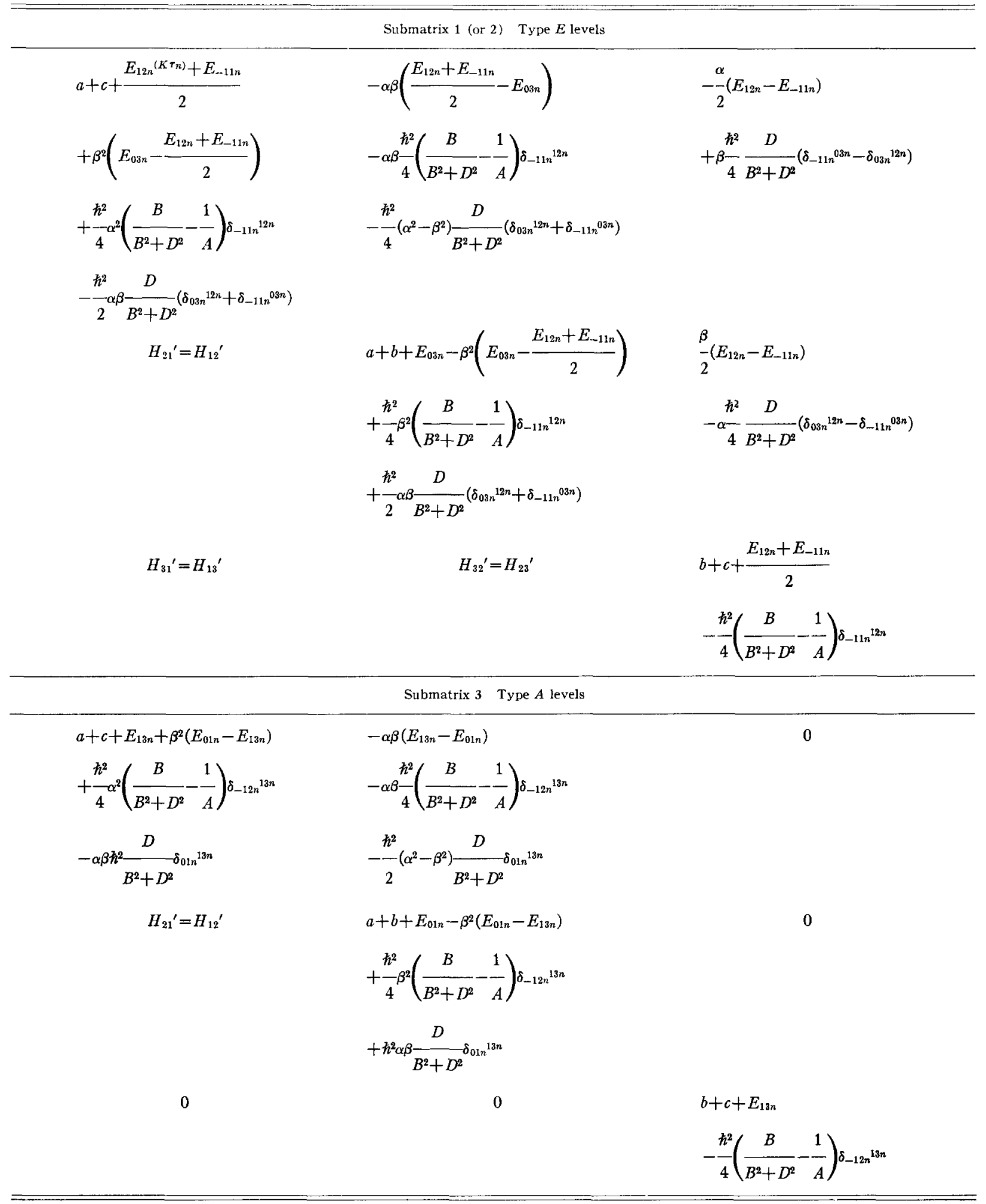

(Eqs. 14), with $\alpha^{2}+\beta^{2}=1$. Their elements can be determined for any $J$ value by the following procedure.

The components of the total angular momentum with respect to principal axes of inertia of the rigid molecule, denoted by $P_{i}{ }^{p}$, are given in terms of the components with respect to the molecule-fixed coordi- 
nate system defined by (6), and denoted by $P_{i}^{\prime}$, by the following equations

$$
\begin{array}{ll}
P_{x}^{p}=P_{x}^{\prime} & P_{y}^{p}=\alpha P_{y}^{\prime}-\beta P_{z}^{\prime} \\
P_{z}^{p}=\beta P_{y}^{\prime}+\alpha P_{z}^{\prime} .
\end{array}
$$

The symmetric rigid rotator wave functions which are solutions of part I of the Hamiltonian in the nonprincipal axis form, (9), will be denoted by $\psi_{J K}$, where $K$ can take on the $2 J+1$ values from $K=-J$ to $K=+J$. (The quantum number $M$ can be suppressed.) The symmetric top wave functions which are solutions of the corresponding part of the Hamiltonian in principal axis form will be denoted by $\psi_{J K_{p}}$, where $K_{p}$ can again take on the $2 J+1$ values from $K_{p}=-J$ to $K_{p}=+J$. A particular wave function $\psi_{J K_{p}}$ will be a linear combination of the $2 J+1$ wave functions $\psi_{J K}$

$$
\psi_{J K_{p}}=\sum_{K=-J}^{K=+J} a_{K_{p} K} \psi_{J K}
$$

where the numbers $a_{K_{p} K}$ are the matrix elements of $\left(S_{1}\right)^{-1}$. The coefficients $a_{K_{p} K}$ are determined most easily by making use of the properties of the operators $P_{y}{ }^{\prime}$, $P_{z}{ }^{\prime}$ on the one hand and the operators $P_{y}{ }^{p}, P_{z}{ }^{p}$ on the other. In the representation used in this paper

$$
\begin{aligned}
P_{z}^{\prime} \psi_{J K} & =\hbar K \psi_{J K}, \\
P_{y}^{\prime} \psi_{J K}= & =-\frac{\hbar}{2}[(J-K)(J+K+1)]^{\frac{1}{2}} \psi_{J(K+1)} \\
& +\frac{\hbar}{2}[(J+K)(J-K+1)]^{\frac{1}{2}} \psi_{J(K-1)}
\end{aligned}
$$

while similar relations hold for $P_{y}{ }^{p}$ and $P_{z}{ }^{p}$ operating on $\psi_{J_{k}}$. The equation

becomes

$$
P_{z}^{p} \psi_{J K_{p}}=\hbar K_{p} \psi_{J K_{p}}
$$

$$
\begin{aligned}
\left(\beta P_{y}^{\prime}+\alpha P_{z}^{\prime}-\hbar K_{p}\right)\left(a_{K_{p}} J \psi_{J J}\right. & +a_{\left.K_{p(J-1}\right)} \psi_{J(J-1)} \\
& \left.+a_{K_{p(J-2)}} \psi_{J(J-2)}+\cdots\right)=0 .
\end{aligned}
$$

Since the $\psi_{J K}$ are linearly independent, the coefficient of each wave function $\psi_{J K}$ in this equation must be equal to zero, leading to the $2 J+1$ equations

$$
\begin{aligned}
& \left(\alpha J-K_{p}\right) a_{K_{p} J}+\frac{\beta}{2}(2 J)^{\frac{1}{2}} a_{K_{p}(J-1)} \\
& \underset{-}{\beta}(2 J)^{\frac{1}{2}} a_{K_{p}}+\left[\alpha(J-1)-K_{p}\right] a_{K_{p}(J-1)}+\frac{\beta}{2}[2(2 J-1)]^{\frac{1}{1}} a_{K_{p}(J-2)} \quad=0 \\
& \stackrel{\beta}{-[2(2 J-1)]^{\frac{1}{2}} a_{K_{p}(J-1)}+\left[\alpha(J-2)-K_{p}\right] a_{K_{p}(J-2)}++_{2}^{-}[3(2 J-2)]^{\frac{1}{1}} a_{K_{p}(J-3)}=0} \\
& \cdot=0
\end{aligned}
$$

From these equations the $a_{K_{p} K}$ can be determined. The signs are chosen so that $S_{1}$ goes over to the identity matrix in the limit $\beta \rightarrow 0, \alpha \rightarrow 1$. Thus, the matrix $S_{1}$ can in principle be found for any $J$ value, and since the matrices $S_{2}$ can be considered to be known, the problem of the asymmetric hindered rotator with relatively high barriers can be reduced to a fairly simple perturbation problem. While this can be done for arbitrarily high $J$ values when $J \geqslant 4$, the analysis must be done numerically.

\section{HIGH BARRIER APPROXIMATIONS} EVALUATION OF THE PERTURBATION TERMS

As has been seen the matrix elements for each particular $J$ value can be reduced to a form in which the offdiagonal elements automatically go to zero as the barrier height goes to infinity. The differences $\left(E_{K \tau n}-E_{K \tau^{\prime} n}\right)$ and the quantities $\delta_{K} K^{\prime \prime}{ }_{\tau}{ }^{r^{\prime}}{ }^{n^{\prime}}$ are small perturbation terms if the hindering barrier is high but finite. The effect of the hindered rotation makes itself felt in two ways. The hindered rotation will cause a splitting of each rigid rotator level into two components since the roots of determinant 3 will differ from those of de- terminant 1 (or 2 ) by small perturbation terms. In addition the center of gravity of the two hindered rotation levels may be shifted slightly from the position of the limiting rigid rotator level. The splittings must be a characteristic of the tunneling effect associated with the hindering barrier and will therefore have considerable physical significance since the barrier height can be deduced from them. Less significance can be attributed to the shifts of the levels, however, since these may be affected much more by all the other vibrationrotation perturbations in the molecule.

It is possible to give relatively simple formulas for the hindered rotation splittings since the perturbation terms given by the $\delta_{K^{K}}{ }^{K^{\prime}}{ }_{\tau^{\prime}}{ }_{n}{ }^{n}$ and the differences $\left(E_{K \tau n}-E_{K \tau^{\prime} n}\right)$ can be approximated by simple expressions in the high barrier case.

In the high barrier limit the $\delta_{K}{ }^{(K+1)}{ }_{\tau^{\tau^{\prime}}{ }_{n}{ }^{n}}$ and the $\delta_{K}{ }^{(K+2)} \tau^{\prime \prime}{ }_{n}{ }^{n}$ turn out, to a very good approximation, to be independent of the particular values of $K$ and $\tau$, and consequently they do not contribute to the splittings of the energy levels at all since the terms occurring in the two types of determinants are identical except for the differences in the $\tau$ values. 
For the $n=0$ to $n=0$ elements :

$$
\begin{aligned}
\delta_{K}{ }^{K+2}{ }_{\tau}{ }^{\prime \prime \prime}{ }_{0}{ }^{0} & \\
= & \frac{2}{3} \frac{1}{\left(H^{\prime}\right)^{2}}\left[1-\left(\frac{C_{1}}{C}\right)^{*}\right]^{2} \\
& +\frac{4}{9} \frac{1}{H^{\prime}}\left\{\left[\frac{9}{8}\left[1-\left(\frac{C_{1}}{C}\right)^{*}\right]^{2}-\frac{1}{2}\left[1-\left(\frac{C_{1}}{C}\right)^{*}\right]^{4}\right\}+\cdots\right.
\end{aligned}
$$$$
\delta_{K}{ }^{K+1} \tau^{\tau^{\prime}{ }_{0}{ }^{0}}
$$$$
\begin{aligned}
= & \frac{1}{6} \frac{1}{\left(H^{\prime}\right)^{\frac{1}{3}}}\left[1-\left(\frac{C_{1}}{C}\right)^{*}\right]^{2} \\
& +\frac{1}{9} \frac{1}{H^{\prime}}\left\{\frac{9}{8}\left[1-\left(\frac{C_{1}}{C}\right)^{*}\right]^{2}-\frac{1}{8}\left[1-\left(\frac{C_{1}}{C}\right)^{*}\right]^{4}\right\}+\cdots
\end{aligned}
$$

in which

and where

$$
H^{\prime}=H / \frac{\hbar^{2}}{2}\left(\frac{C}{C_{1} C_{2}}\right)^{*},
$$

$$
\frac{\hbar^{2}}{2}\left(\frac{C}{C_{1} C_{2}}\right)^{*}=\frac{\hbar^{2}}{2} \frac{\left(B C-D^{2}\right)}{C_{2}\left(B C_{1}-D^{2}\right)}
$$

is the natural energy unit for measuring the height of the barrier, $H$.

The form of the internal rotation energy is also a relatively simple one in the high barrier limit. The internal rotation energy must be a periodic function of $\gamma=(2 \pi / 3) K\left(C_{1} / C\right)^{*}$, and hence may be expanded as a Fourier series in $\gamma$. In the high barrier limit, however, only the first term in the Fourier series is significant and it can be shown that the higher terms in the series are completely negligible. Therefore

$$
\begin{gathered}
E_{K 1 n}=E_{n}-\frac{2}{3} \Delta_{0}{ }^{(n)} \cos \gamma, \\
\left\{\begin{array}{l}
E_{K 2 n} \\
E_{K 3 n}
\end{array}\right\}=E_{n}-\frac{2}{3} \Delta_{0}^{(n)} \cos \left(\gamma \pm \frac{2 \pi}{3}\right),
\end{gathered}
$$

where $E_{n}$ is given by

$$
\begin{aligned}
E_{n}= & \frac{\hbar^{2}}{2}\left(\frac{C}{C_{1} C_{2}}\right)^{*}\left\{3\left(n+\frac{1}{2}\right)\left(H^{\prime}\right)^{\frac{1}{2}}\right. \\
& -\frac{9}{8}\left[\left(n+\frac{1}{2}\right)^{2}+\frac{1}{4}\right]-\frac{27}{64} \frac{1}{\left(H^{\prime}\right)^{\frac{1}{2}}}\left[\left(n+\frac{1}{2}\right)^{3}+\frac{3}{4}\left(n+\frac{1}{2}\right)\right] \\
& \left.-\frac{3^{4}}{2^{10}} \frac{1}{H^{\prime}}\left[5\left(n+\frac{1}{2}\right)^{4}+\frac{17}{2}\left(n+\frac{1}{2}\right)^{2}+\frac{9}{16}\right]+\cdots\right\},
\end{aligned}
$$

and where $\Delta_{0}{ }^{(n)}$, the splitting of the energy levels of the simple hindered rotator, in the $n$th torsional state, for $K=0$, can be given as a fairly simple function of the parameter $H^{\prime}$.
One of the best methods of estimating the differences $\left(E_{K \tau n}-E_{K \tau^{\prime} n}\right)$ is through the use of the WKB approximation. The values of $\delta_{K} K^{\prime}{ }_{\tau}{ }^{\prime}{ }_{n}{ }^{n^{\prime}}$ and $E_{n}$, on the other hand, are given to very good approximation by ordinary perturbation theory, using as zeroth-order wave functions the correct linear combinations of harmonic oscillator functions centered at the three potential minima.

\section{WKB Approximation}

The splitting of the energy levels for the simple hindered rotator with nonperiodic boundary conditions $(K \neq 0)$ can be estimated by means of a straightforward application of the WKB approximation by a slight extension of the methods of Koenig. ${ }^{10}$ In first approximation the results are given by Eqs (18). The WKB value for $\Delta_{0}{ }^{(n)}$, the $K=0$ splitting of the energy levels for the simple hindered rotator, in the $n$th torsional state, is given by

in which

$$
\Delta_{0}(n)^{\prime}=\frac{9}{4}\left(H^{\prime}\right)^{\frac{3}{3}} \frac{e^{-2 I}}{K\left(k^{2}\right)},
$$

$$
I=\frac{2}{3}\left(H^{\prime}\right)^{\frac{1}{2}}\left[E\left(1-k^{2}\right)-k^{2} K\left(1-k^{2}\right)\right],
$$

and where $K$ and $E$ are complete elliptic integrals of the first and second kinds, with $k^{2}=\left(E_{n} / H\right)$. All primed quantities are measured in the natural energy unit, $\hbar^{2} / 2\left(C / C_{1} C_{2}\right)^{*}$.

The complete WKB expression for the splittings of the energy levels will contain the higher terms of the Fourier series, for example, terms such as $\cos 2 \gamma$, $\cos 3 \gamma, \cdots$, for $\tau=1$; but the coefficients of these terms will be of order $e^{-4 I}, e^{-6 I}, \cdots$, respectively, and may thus be expected to be completely negligible compared with the coefficient of the dominant first term since the exponential $e^{-2 I}$ gives the probability of penetration of the barrier. This follows not only from the results of the WKB approximation but is borne out also by exact sample calculations.

Since it is our aim to determine the barrier height from the observed splittings this form of the splitting formula, (20), is not a very useful one since the functional relationship between $\Delta_{0}{ }^{(n)}$ and $H$ is very complicated. The explicit dependence of $\Delta_{0}{ }^{(n)}$ on the barrier height can be seen more easily if the quantities in $\mathrm{Eq}$. (20) are expanded in powers of $E_{n} / H$, leading to the expression

$$
\begin{aligned}
\Delta_{0}^{(n)^{\prime}}=\frac{9}{2 \pi}\left[\frac{16}{3(n+1 / 2)}\right]^{n+1 / 2} e^{n+1 / 2} H^{\prime[(3 / 4)+(n / 2)]} \\
\times e^{-(4 / 3)\left(H^{\prime}\right) !}\left[1+\text { order } H^{\prime-\frac{1}{2}}\right] .
\end{aligned}
$$

However, neither formula (20) nor (21) gives a very accurate estimate of the hindered rotation splittings of the energy levels. Sample calculations for certain values

\footnotetext{
${ }^{10}$ H. D. Koenig, Phys. Rev. 44, 657 (1933).
} 
of $H^{\prime}$ in the region of present interest, for which $50 \leqslant H^{\prime} \leqslant 200$ (as will be shown in a later section) indicate that the WKB expressions for $\Delta_{0}{ }^{(n)^{\prime}}$ may be in error by as much as ten to twenty percent. However Eq. (21) is very useful since it can form the basis for a semiempirical expression for $\Delta_{0}(n)^{\prime}$ by which the correct values for $\Delta_{0}{ }^{(n)}$ ', computed by the continued fractions technique, ${ }^{1}$ can be fitted with considerable accuracy. For $n=0$, a semiempirical formula will be chosen having the form

$$
\Delta_{0}^{\prime}=a H^{\prime 1} e^{-b \vee H^{\prime}},
$$

in which the two constants $a$ and $b$ are now determined to give the best possible fit to the correct values for $\Delta_{0}{ }^{\prime}$. The following formula has been found to be useful and can be expected to be accurate to within one or two percent for the range $50 \leqslant H^{\prime} \leqslant 200$

$$
\Delta_{0}{ }^{\prime}=7.05 H^{\prime \frac{3}{3}} e^{-1.324 \sqrt{ } H^{\prime}} .
$$

\section{Perturbation Theory}

Approximate expressions for the $\delta_{K} K^{\prime}{ }^{\tau^{\tau^{\prime}}{ }^{n^{\prime}}}$ and the values of $E_{n}$ are obtained most simply by means of standard perturbation theory involving only the internal rotation wave functions

$$
M(x)=e^{-i K\left(C_{1} / C\right)^{*} x} P_{K \tau n}(x) .
$$

Since the hindering potential is a quadratic function of $x$ in the immediate vicinity of the three potential minima, the zeroth-order wave functions are linear combinations of harmonic oscillator functions centered at the three minima and therefore very simple in form. The linear combinations having the appropriate symmetry for a threefold potential have been given in the paper by Koehler and Dennison. In the limit of a truly infinite barrier the three types of wave functions are given equally well by the single formula

$$
\begin{array}{r}
P_{K \tau n}(x)=\frac{1}{\sqrt{3}}\left\{H_{n}^{(1)}(x)+H_{n}^{(2)}(x)+H_{n}{ }^{(3)}(x)\right\} e^{i \rho x} \\
=u_{n}(x) e^{i \rho x},
\end{array}
$$

where $H_{n}{ }^{(i)}(x)$ is the harmonic oscillator wave function centered at the $i$ th minimum, for which $n$ is now the ordinary vibrational quantum number, and where

$$
\begin{array}{lll}
\rho=0+3 l & \text { for }(K-3 l)=-1, \quad 0,+1 & \text { when } \tau=1 \\
\rho=-1+3 l & \text { for }(K-3 l)=0,-1,-2 & \text { when } \tau=2 \\
\rho=+1+3 l & \text { for }(K-3 l)=0,+1,+2 & \text { when } \tau=3
\end{array}
$$

with $l$ taking on all integral values. These wave functions form a convenient set of zeroth-order wave functions for the perturbation problem in the high barrier case. The internal rotation wave functions $M(x)$ must be eigenfunctions of the simple hindered rotator
Hamiltonian

$$
\mathfrak{C}=-\frac{\hbar^{2}}{2}\left(\frac{C}{C_{1} C_{2}}\right)^{*} \frac{\partial^{2}}{\partial x^{2}}+\frac{H}{2}(1-\cos 3 x),
$$

but in terms of the simpler functions, $u_{n}(x)$, the wave equation is given by

$$
\begin{aligned}
\left\{\left(\mathfrak{H}^{(0)}+\mathscr{K}^{(1)}+\mathcal{H}^{(2)}+\cdots\right)-\left(E_{n}{ }^{(0)}+E_{n}{ }^{(1)}+E_{n}{ }^{(2)}+\cdots\right)\right\} \\
\\
\times\left\{u_{n}{ }^{(0)}(x)+u_{n}{ }^{(1)}(x)+u_{n}{ }^{(2)}(x)+\cdots\right\}=0,
\end{aligned}
$$

where, for the three regions, $\left|x-x_{0}{ }^{(i)}\right| \leqslant \pi / 3$

$\mathfrak{F}^{(0)}=-\frac{\hbar^{2}}{2} \frac{\partial^{2}}{\partial x^{2}}\left(\frac{C}{C_{1} C_{2}}\right)^{*}+\frac{9}{4} H\left(x-x_{0}{ }^{(i)}\right)^{2}$
$\quad$ of order $\left.H^{3}\right\}$

$\mathfrak{H}^{(1)}=\frac{2}{i} \frac{\hbar^{2}}{2}\left(\frac{C}{C_{1} C_{2}}\right)^{*}\left[\rho-K\left(\frac{C_{1}}{C}\right)^{*}\right]_{\partial x}^{\frac{\partial}{\partial x}}$

\{of order $\left.H^{\frac{1}{4}}\right\}$

$\mathfrak{F}^{(2)}=\frac{\hbar^{2}}{2}\left(\frac{C}{C_{1} C_{2}}\right)^{*}\left[\rho-K\left(\frac{C_{1}}{C}\right)^{*}\right]^{2}$

$$
-\frac{27}{16} H\left(x-x_{0}^{(i)}\right)^{4} \quad \text { of order } 1 \text { \} }
$$

$\mathfrak{F}^{(3)}=0$

$\mathfrak{H}^{(4)}=\frac{81}{160} H\left(x-x_{0}{ }^{(i)}\right)^{6}$

\{of order $H^{-\frac{1}{2}}$ \}

where $\mathcal{F}^{(0)}$ gives contributions to the energy of order $\left(H^{\frac{1}{3}}\right)$, etc.

In this perturbation scheme the wave functions $u_{n}(x)$ and the energies $E_{n}{ }^{11}$ can be determined up to any order by nondegenerate perturbation theory with the use of the zeroth-order wave functions $u_{n}^{(0)}(x)$. The calculations are simplified by the use of the following further high barrier approximations which can be expected to be very good for values of $H^{\prime} \geqslant 50$. The wave functions $H_{n}{ }^{(i)}(x)$ will be assumed to be negligible for $\left|x-x_{0}{ }^{(i)}\right| \geqslant \pi / 3$ so that all integrals involving the $H_{n}{ }^{(i)}(x)$ can be carried out from $-\infty$ to $+\infty$, and overlap integrals of the type $\int H_{n}{ }^{(1)}(x) \mathcal{O} H_{n}{ }^{(2)}(x) d x$ can be neglected. It is because of these approximations that the results for the $\delta_{K} K^{\prime}{ }_{\tau}{ }^{\gamma^{\prime}}{ }_{n}{ }^{\prime}$ given in Eq. (17) are independent of $\tau$ and $K$. Further approximations would have to take into account the proper connections of the wave functions in the regions $\left(x-x_{0}{ }^{(i)}\right)=\pi / 3$; but actual numerical calculations indicate that such further approximations are not needed for an accurate determination of the barrier height.

${ }^{11}$ For the $E_{n}$, see also, Tables Relating to Mathieu Functions (Columbia University Press, New York, 1951), p. XVIII. 
With the use of these approximations the matrix elements of the Hamiltonian (26) become

$$
\begin{aligned}
& H_{(n-1) n}{ }^{(\mathrm{l})}=-i \hbar \omega\left[\frac{4}{9 H^{\prime}}\right]^{\frac{1}{}}\left[\rho-K\left(\frac{C_{1}}{C}\right)^{*}\right]\left(\frac{n}{2}\right)^{\frac{1}{2}}, \\
& H_{n n}{ }^{(2)}=\hbar \omega\left[\frac{4}{9 H^{\prime}}\right]^{\frac{1}{2}} \\
& \times\left\{\frac{1}{2}\left[\rho-K\left(\frac{C_{1}}{C}\right)^{*}\right]^{2}-\frac{9}{16}\left(n^{2}+n+\frac{1}{2}\right)\right\}, \\
& H_{n(n-2)}(2)=-\hbar \omega\left[\frac{4}{9 H^{\prime}}\right]^{\frac{1}{2}}\left\{\frac{3}{16}(2 n-1)[n(n-1)]^{3}\right\}, \\
& H_{n(n-4)^{(2)}}=-\hbar \omega\left[\frac{4}{9 H^{\prime}}\right]^{\frac{1}{2}} \\
& \times\left\{\frac{3}{32}[n(n-1)(n-2)(n-3)]^{\frac{1}{3}}\right\}, \\
& H_{n n}{ }^{(4)}=\hbar \omega\left[\frac{4}{9 H^{\prime}}\right]\left\{\frac{9}{32}\left[\left(n+\frac{1}{2}\right)^{3}+(5 / 4)\left(n+\frac{1}{2}\right)\right]\right\} .
\end{aligned}
$$

With $E_{n}{ }^{(0)}=\hbar \omega\left(n+\frac{1}{2}\right) ; \hbar \omega=3 \sqrt{ } H^{\prime}\left(\hbar^{2} / 2\right)\left(C / C_{1} C_{2}\right)^{*}$. Since the parameter of smallness in the perturbation scheme is given by $\left(4 / 9 H^{\prime}\right)^{\frac{1}{4}}$ the wave functions $u_{n}(x)$ must be computed up to fourth order in order to obtain accurate values for the $\delta_{K} K^{\prime}{ }^{\prime} \tau^{\prime}{ }_{n}^{n}$ for barriers for which $H^{\prime} \geqslant 50$. The wave functions $u_{n}(x)$ are functions of $\rho$ and $K$, but the structure of the $K$ to $K^{\prime}$ connections in the Hamiltonian and the symmetries of the wave functions

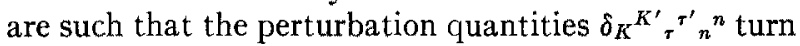
out to be independent of $\rho$ and $K$ and are given by Eqs. (17) for $n=0$.

To within the approximations made in this section therefore the perturbation terms $\delta_{K} K^{\prime}{ }_{r} r^{\gamma^{\prime}}{ }_{n}{ }^{n}$ contribute only to the shift of the center of gravity of two hindered rotation sublevels from the position of the limiting rigid rotator level. Since the quantities of greatest interest are the hindered rotation splittings, not the shifts, the possibility exists that the small errors inherent in these approximations may lead to small errors in the determination of the barrier height. However, actual numerical calculations in some special cases show that the small differences in the $\delta_{K}{ }^{K^{\prime}}{ }_{r}{ }^{\prime}{ }_{n}{ }^{n}$ for different values of $\tau$ are indeed negligible as far as their contribution to the hindered rotation splittings is concerned and that Eqs. (17) can be expected to be accurate to within a few percent for values of $H^{\prime} \geqslant 50$.

However, since the overall shifts of the levels may affect the determination of the principal moments of inertia, it becomes a matter of some importance to know their order of magnitude. The hindered rotation shifts are determined not only by the magnitudes of the $\delta_{K}{ }^{K}{ }_{r}{ }^{\prime}{ }_{n}^{n}$ but also by the magnitudes of the asymmetry parameters

$$
\frac{h}{8 \pi^{2}}\left(\frac{1}{A}-\frac{B}{B^{2}+D^{2}}\right) \text { and } \frac{h}{8 \pi^{2}} \frac{D}{B^{2}+D^{2}}
$$

However, even for very large values of these asymmetry parameters, $30000 \mathrm{Mc} / \mathrm{sec}$ for example, the hindered rotation shifts can be expected to be of the same order of magnitude as the hindered rotation splittings of the energy levels.

Consequently, if the hindered rotation splittings of the levels have been found to be small enough, the principal moments of inertia can be determined with fair accuracy from the average positions of the two hindered rotation lines of the rotational transitions. Once the barrier height has been determined with these values of the moments of inertia, the hindered rotation shifts of the levels can be computed, and the cycle of calculations can be repeated in order to improve the accuracy of the values for the moments of inertia. However, since the values will be affected by all the other vibration perturbations no specific formulas for the hindered rotation shifts will be tabulated. In principle these shifts can be computed for any level with considerable accuracy since the $\delta_{K^{K}}{ }^{K^{\prime}}{ }_{\tau}{ }^{\prime}{ }_{n}{ }^{n}$, computed to order $\left(4 / 9 H^{\prime}\right)$, have been found to be accurate to within a few percent. In order to predict the shifts with this accuracy, however, the $n \rightarrow n^{\prime}\left(n^{\prime} \neq n\right)$ connections can no longer be neglected. These are of order $\left(4 / 9 H^{\prime}\right)^{t}$, and since the hindered rotation energies are of order $\left(9 H^{\prime} / 4\right)^{z}$, the $n \rightarrow n^{\prime}$ connections give contributions to the rotational energies of the order $\left(4 / 9 H^{\prime}\right)$. For the $n=0 \rightarrow 1$ connections, for example

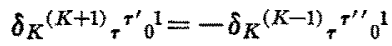

$$
\begin{aligned}
& =-\frac{i}{\sqrt{2}}\left[\frac{4}{9 H^{\prime}}\right]^{\frac{i}{3}}\left[1-\left(\frac{C_{1}}{C}\right)^{*}\right], \\
& \delta_{K}{ }^{(K+2)}{ }_{\tau}^{\tau^{\prime \prime}} 0_{0}^{1}=-\delta_{K}{ }^{(K-2)} \tau_{\tau}{ }^{\prime} 0^{1} \\
& =-i \sqrt{2}\left[\frac{4}{9 H^{\prime}}\right]^{\frac{1}{1}}\left[1-\left(\frac{C_{1}}{C}\right)^{*}\right] .
\end{aligned}
$$

The effect of these terms, off-diagonal in the quantum number $n$, can be brought into the rotational matrix elements, diagonal in $n$, by the Van Vleck perturbation technique. The transformed $n \rightarrow n^{\prime}$ matrix elements can then be neglected, and the rotational matrix elements diagonal in $n$, which now give the energies correct to order $\left(4 / 9 H^{\prime}\right)$ will be given by the usual expression. ${ }^{12}$

${ }^{12}$ See, for example, E. B. Wilson and J. B. Howard, J. Chem. Phys. 4, 260 (1936), Eq. (43). 
For $n=0$ they are

$$
\begin{aligned}
& H_{J K \tau 0}{ }^{J K \tau}{ }^{2}=\frac{\hbar^{2}}{4}\left(\frac{1}{A}+\frac{B}{B^{2}+D^{2}}\right)\left(J^{2}+J-K^{2}\right)+\frac{\hbar^{2}}{2}\left(\frac{B+C}{B C-D^{2}}-\frac{B}{B^{2}+D^{2}}\right) K^{2}+E_{K \tau n}{ }^{\text {int }} \\
& -\frac{1}{9} \frac{1}{H^{\prime}}\left[1-\left(\frac{C_{1}}{C}\right)^{*}\right]^{2}\left\{\frac { \hbar ^ { 2 } } { 4 } ( \frac { C _ { 2 } C _ { 1 } } { C } ) ^ { * } \left[( \frac { 1 } { A } - \frac { B } { B ^ { 2 } + D ^ { 2 } } ) ^ { 2 } \left\{J^{2}(J+1)^{2}+K^{2}\left(K^{2}+5\right)\right.\right.\right. \\
& \left.\left.\left.-2 J(J+1)\left(K^{2}+1\right)\right\}+\left(\frac{D}{B^{2}+D^{2}}\right)^{2}\left\{J(J+1)\left(4 K^{2}+1\right)-K^{2}\left(4 K^{2}+5\right)\right\}\right]\right\}, \\
& H_{J K \tau 0} J(K+1) \tau^{\prime} 0=\frac{h^{2}}{4} \frac{D}{B^{2}+D^{2}}(2 K+1)[(J-K)(J+K+1)]^{\frac{1}{2}} \\
& \times\left\{1-\frac{1}{6} \frac{1}{\sqrt{ } H^{\prime}}\left[1-\left(\frac{C_{1}}{C}\right)^{*}\right]^{2}-\frac{1}{9} \frac{1}{H^{\prime}}\left(\frac{9}{8}\left[1-\left(\frac{C_{1}}{C}\right)^{*}\right]^{2}-\frac{1}{8}\left[1-\left(\frac{C_{1}}{C}\right)^{*}\right]^{4}\right)\right\} \\
& +\frac{1}{9} \frac{1}{H^{\prime}}\left[1-\left(\frac{C_{1}}{C}\right)^{*}\right]^{2} \frac{\hbar^{2}}{4}\left(\frac{C_{2} C_{1}}{C}\right)^{*} \frac{D}{B^{2}+D^{2}}\left[\frac{1}{A}-\frac{B}{B^{2}+D^{2}}\right] \\
& \times\left\{(2 K+1)[J(J+1)-K(K+1)-3][(J-K)(J+K+1)]^{1}\right\}, \\
& \mathrm{H}_{J K \tau 0}{ }^{J(K+2) \tau^{\prime \prime} 0}=-\frac{\hbar^{2}}{8}\left(\frac{1}{A}-\frac{B}{B^{2}+D^{2}}\right)[(J-K)(J-K-1)(J+K+1)(J+K+2)]^{\frac{1}{3}} \cdot\left[1-\frac{2}{3} \frac{1}{\sqrt{ } H^{\prime}}\left[1-\left(\frac{C_{1}}{C}\right)^{*}\right]^{2}\right. \\
& \left.-\frac{4}{9}\left\{\frac{9}{8}\left[1-\left(\frac{C_{1}}{C}\right)^{*}\right]^{2}-\frac{1}{2}\left[1-\left(\frac{C_{1}}{C}\right)^{*}\right]^{4}\right\}\right]+[(J-K)(J-K-1)(J+K+1)(J+K+2)]^{\frac{1}{3}} \\
& \times\left\{\frac{1}{9} \frac{1}{H^{\prime}} \frac{h^{2}}{8}\left(\frac{C_{2} C_{1}}{C}\right)^{*}\left[1-\left(\frac{C_{1}}{C}\right)^{*}\right]^{2} \cdot \frac{D^{2}}{B^{2}+D^{2}}(2 K+1)(2 K+3)\right\} \\
& H_{J K r 0}{ }^{J(K+3) \tau 0}=-\frac{1}{9} \frac{1}{H^{\prime}}\left[1-\left(\frac{C_{1}}{C}\right)^{*}\right]^{2} \frac{\hbar^{2}}{4}\left(\frac{C_{2} C_{1}}{C}\right)^{*} \frac{D}{B^{2}+D^{2}}\left(\frac{1}{A}-\frac{B}{B^{2}+D^{2}}\right) \\
& \cdot(2 K+3)[(J-K)(J-K-1)(J-K-2)(J+K+1)(J+K+2)(J+K+3)]^{\frac{1}{2}}, \\
& H_{J K \tau}{ }^{J(K+4) r^{\prime} 0}=\frac{1}{9} \frac{1}{H^{\prime}}\left[1-\left(\frac{C_{1}}{C}\right)^{*}\right]^{2} \frac{h^{2}}{8}\left(\frac{C_{2} C_{1}}{C}\right)^{*}\left(\frac{1}{A}-\frac{B}{B^{2}+D^{2}}\right)^{2} \\
& \cdot\left[(J-K)(J-K-1)(J-K-2)(J-K-3)(J+K+1)(J+K+2)(J+K+3)(J+K+4]^{\sharp}\right) \text {. }
\end{aligned}
$$

\section{HINDERED ROTATION SPLITTINGS OF THE} ASYMMETRIC RIGID ROTATOR LEVELS

Since the value of the barrier height can be deduced from the hindered rotation splittings these are the quantities of greatest interest and specific formulas for them will be tabulated in this section for some of the low $J$ values. The matrix elements in the form of Table I can be used even if the asymmetry splitting of a pair of rigid rotator levels is of the same order of magnitude as the hindered rotation splittings, but in that case the formulas for the energies are very complicated. Simple splitting formulas can be given, however, if the hindered rotation splittings of the levels are small compared to the asymmetry splittings. In that case the hindered rotation splits the rigid rotator level $J_{K_{-1} K_{1}}$ into two sublevels, one, $J_{K_{-1} K_{1}}{ }^{E}$ (of species $E$ ), and one, $J_{K_{-1} K_{1}{ }^{A}}$ (of species $A$ ). The hindered rotation splittings are given for some of the low $J$ values in Table II. However, these formulas may not apply to certain levels of a particular molecule, since the asymmetry splittings for certain of the levels may be comparable to the hindered rotation splittings.

The labeling of the levels in Table II is that for which the magnitudes of the principal moments of inertia are such that $a<b<c$. The relative magnitudes of the principal moments of inertia do not affect the splitting formulas but only the labeling of the energy levels. Thus, the rigid rotator level whose energy is given by $a+b+4 c$, for example, will show a hindered rotation splitting $\Delta_{2}+\beta^{2}\left(\Delta_{1}-\Delta_{2}\right)$, but for $a<b<c$ this will be the splitting formula for the level $2_{21}$, whereas for $a<c<b$ this would have been the splitting formula for the level $2_{11}$, etc. Since the $x, y$, and $z$ axes of the molecular framework of the hindered rotator do not correspond to equivalent directions, the moment of inertia $A$, for example, cannot arbitrarily be chosen to be the greatest or least moment of inertia.

It can be seen that the splitting formulas are func- 
TABLE II. Hindered rotation splittings of the asymmetric rigid rotator levels.

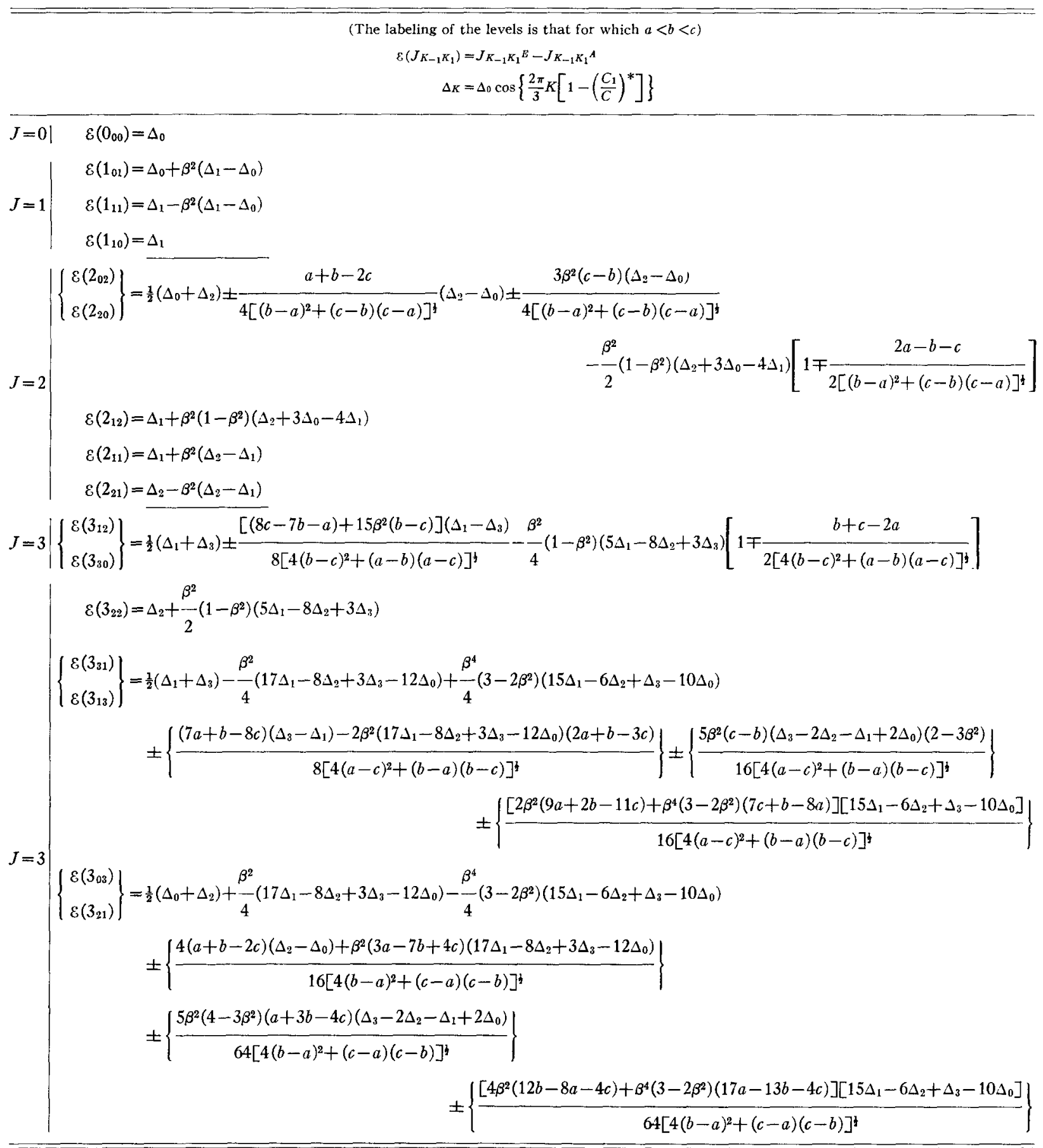

tions only of the principal moments of inertia and the three additional molecular parameters

$$
\Delta_{0},\left(\frac{C_{1}}{C}\right)^{*}, \text { and } \beta^{2}
$$

The parameters $\left(C_{1} / C\right)^{*}$ and $\beta^{2}$ depend only on the mass distribution and the geometry of the molecule. $\beta^{2}$ goes to zero in the limit in which the $z$-principal axis coincides with the symmetry axis of the hindering po- tential. For small values of the product of inertia, $D$, $\beta^{2} \approx D^{2} C^{2} / B^{2}(B-C)^{2}$. The splitting parameter, $\Delta_{0}$, on the other hand is related to the barrier height, by formula (23) for example.

If the principal moments of inertia are known with fair accuracy from an analysis of the gross features of the spectrum, the three additional molecular parameters can be determined if three different line splittings have been observed. Further, if the principal moments of inertia are known, the value of $C_{1}$ can be determined 
from $\left(C_{1} / C\right)^{*}$, the value of the product of inertia $D$ from $\beta^{2}$, and all the information needed is at hand since the barrier height can be determined from $\Delta_{0}$ by means of formula (23) once the energy unit $\hbar^{2} / 2\left(B C-D^{2}\right) /$ $C_{2}\left(B C_{1}-D^{2}\right)$ has been computed from the molecular parameters.

\section{SYMMETRY PROPERTIES; ORDERING OF THE TYPE A LEVELS; SELECTION RULES}

The symmetry properties for the more symmetrical hindered rotators of the nitromethane type have been examined by Wilson, Lin, and Lide. ${ }^{6}$ For the asymmetric hindered rotator of present interest the Hamiltonian will be invariant under the following symmetry operations: the identity operation, $(I)$; the two threefold rotations of the symmetric component of the molecule about its symmetry axis, $\left(C_{3}, C_{3}^{2}\right.$, or $\left.\phi_{2} \rightarrow \phi_{2} \pm 2 \pi / 3\right)$; a twofold rotation of the whole molecule about the $x$ axis, the axis perpendicular to the plane of symmetry of the asymmetric component of the molecule, $\left(C_{2 x}\right.$ or $\left.\theta \rightarrow \pi-\theta, \psi \rightarrow \psi+\pi, \phi_{1} \rightarrow \pi-\phi_{1}, \phi_{2} \rightarrow \pi-\phi_{2}\right)$, and finally the operations $C_{2 x} C_{3}$ and $C_{2 x} C_{3}{ }^{2}$. These symmetry operations satisfy the group properties of the group $D_{3}$, and the energy levels will therefore fall into species $A_{1}$, $A_{2}$, or $E$. The symmetry elements $I$ and $C_{2 x}$ form a subgroup of both the hindered rotator and the asymmetric rigid rotator symmetries, and from the compatibility relations it can be seen that each rigid rotator level must give rise to one level of species $E$ and one of species $A$. The wave functions for the type $A$ levels have one of two possible forms. These have been given in the paper by Ivash and Dennison ${ }^{3}$ and have been labeled + and - . However, the Ivash-Dennison + or - refers to the particular structure of the wave function, not to a change of sign of the wave function under a symmetry operation. If their \pm is multiplied by $(-1)^{J+n}$ the resulting \pm refers to the change of sign of the wave function under the symmetry operation $C_{2 x}$ where the new + levels are then the $A_{1}$ levels, the new - levels the $A_{2}$ levels.

Since the symmetry operation $C_{2 x}$ is common to both the asymmetric rigid rotator and the hindered rotator Hamiltonians, the rigid rotator energy levels which have the property + with respect to a twofold rotation about the $x$ axis will give rise to an $A_{1}$ level in the hindered rotator, while the rigid rotator energy levels which have the property - - with respect to such a rotation give rise to an $A_{2}$ level.

In molecules such as methyl alcohol in which the $x$ axis is the axis of the greatest moment of inertia the ordering of the type $A$ levels starting with the highest level of a particular $J$ value is therefore: $A_{1}, A_{2}, A_{2}$, $A_{1}, A_{1}, A_{2}, A_{2}, \cdots$ (for $n=0$ or even).

In a molecule in which the $x$ axis is the axis of the middle moment of inertia, however, the ordering of the $A$ levels would be: $\cdots A_{1}, A_{2}, A_{1}, A_{2}, A_{1}, \cdots$.

The selection rules for this type of asymmetric hindered rotator have been given in the paper by Ivash and Dennison but can be expressed more easily in terms of the symmetry species $A_{1}, A_{2}$, and $E$. They are simply

$$
\begin{array}{cc}
A_{1} \leftrightarrow A_{2} & \Delta J=0, \pm 1 \\
E \leftrightarrow E & \Delta M=0, \pm 1
\end{array}
$$

The $A_{1} \leftrightarrow A_{2}$ transitions will always correspond to transitions which are allowed for the limiting rigid molecule. Some of the $E \leftrightarrow E$ transitions, however, may be forbidden for the limiting rigid molecule and may be expected to appear only weakly in the spectrum. Essentially the spectrum will be that of the rigid rotator, with the important exception that each line of the spectrum is split into two components of comparable intensity, one $A_{1} \leftrightarrow A_{2}$, the other $E \leftrightarrow E$.

\section{LIMITATIONS OF THE HIGH BARRIER APPROXIMATIONS}

So far the term "relatively high potential barrier" has been defined only qualitatively. A molecule with a relatively high barrier to internal rotation is one in which the hindered rotation splittings are in general much less than the separations between the rigid rotator energy levels and yet large enough to be easily observable. Previously it was shown that the high barrier

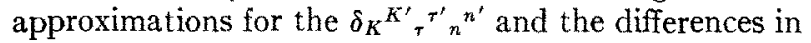
the $E_{K r n}$ are very good for values of $H^{\prime} \geqslant 50$. However, for $H^{\prime} \approx 50$ the hindered rotation splittings may be of the same order of magnitude as the separations between the rigid rotator levels, and the simple splitting formulas of Table II may not apply. In this case the off-diagonal matrix elements as given in Table I can no longer be neglected, and second- or higher-order perturbation terms may be needed in order to get a good estimate of the splittings of the energy levels. In this case also the splittings may be so large that the lines no longer appear as doublets. On the other side of the scale, for $H^{\prime}>200$, the hindered rotation splittings begin to fall below the limits set by the microwave resolution, and thus although the splitting formulas are correct they cannot be tested experimentally.

As stated before, the natural unit for measuring the energy of the asymmetric hindered rotator is given by the quantity $\hbar^{2} / 2\left(C / C_{1} C_{2}\right)^{*}$. Since this depends on the masses of the atoms, a barrier height of $500 \mathrm{~cm}^{-1}$, for example, may be a low barrier for one molecule but may be so high for another molecule that the latter can essentially be regarded as a rigid top. Three types of molecules must be considered. First, a molecule such as methyl alcohol in which all the off-axial atoms of both components of the molecule are hydrogen atoms. For such a molecule the barrier height would have to be greater than $2000 \mathrm{~cm}^{-1}$ if the high barrier approximations are to hold. The second type of molecule is a molecule such as ethylfluoride in which the off-axial atoms of one component of the molecule are all hydrogen atoms while the other component contains at least one heavy off-axial atom. For such a molecule a barrier height greater than about $400 \mathrm{~cm}^{-1}$ is a high one. The third type of molecule is a molecule such as 
$\mathrm{CF}_{3} \mathrm{CH}_{2} \mathrm{~F}$ in which both components contain heavy off-axial atoms. For such a molecule the barrier height would only have to be greater than about $40 \mathrm{~cm}^{-1}$. On the other hand such a molecule could be regarded as a rigid top if the barrier height were greater than about $200 \mathrm{~cm}^{-1}$.

On the basis of the barrier height for methyl alcohol it seems probable that more numerous examples of high barriers in the sense of the present paper will be found among the molecules of the second type than in types one and three. There is, however, one difficulty which may occur. As the ratio $\left(C_{1} / C\right)^{*}$ approaches unity, the splittings of all the energy levels become identical, so that the observed lines are not split at all, even though the splittings of the energy levels may be large. Unfortunately the second type of molecule is just one for which $\left(C_{1} / C\right)^{*}$ may be close to unity. However, even in this case certain of the lines will show a splitting of the order of magnitude of the splittings of the energy levels themselves. Unfortunately these lines may involve high $J$ and $K$ values where both the identifications of the lines and the computations become very difficult. These circumstances may explain why Solimene and Dailey ${ }^{13}$ have not observed any splittings in the higher torsional satellites of the low $J$ transitions in 1,1-di- fluoroethane. The acetaldehyde molecule, however, is one of the second type in which the ratio $\left(C_{1} / C\right)^{*}$ is such that the line splittings should be easily observable.

\section{APPENDIX I. GENERALIZATION TO MORE ASYMMETRIC TYPES OF MOLECULES}

The theory of hindered rotation in molecules with relatively high potential barriers has been given for only a specific, simple type of asymmetric molecule. However, the methods used can be generalized to more asymmetric molecules.

A slightly more complex type is one consisting of a completely asymmetric component linked in an arbitrary manner to a symmetric component. This type of molecule has been treated by Burkhard. ${ }^{14}$ The kinetic energy is

$$
\begin{aligned}
2 T=A \omega_{x}^{2}+B \omega_{y}{ }^{2}+C \omega_{z}{ }^{2}- & -2 D \omega_{y} \omega_{z}-2 E \omega_{x} \omega_{y} \\
& -2 F \omega_{x} \omega_{z}+C_{2} \dot{x}^{2}-2 C_{2} \dot{x} \omega_{z} .
\end{aligned}
$$

The Hamiltonian can be expressed in terms of the total angular momentum components, $P_{i}=\partial T / \partial \omega_{i}$, and the momentum conjugate to $x, p_{x}=\partial T / \partial \dot{x}$. In the high barrier case it is again important to make a transformation to a molecule-fixed system of axes which is now defined by the transformation

$$
\begin{aligned}
& p_{x}=p_{x}{ }^{\prime}-\left(\frac{C_{2}}{C}\right)^{*} P_{z}{ }^{\prime},
\end{aligned}
$$

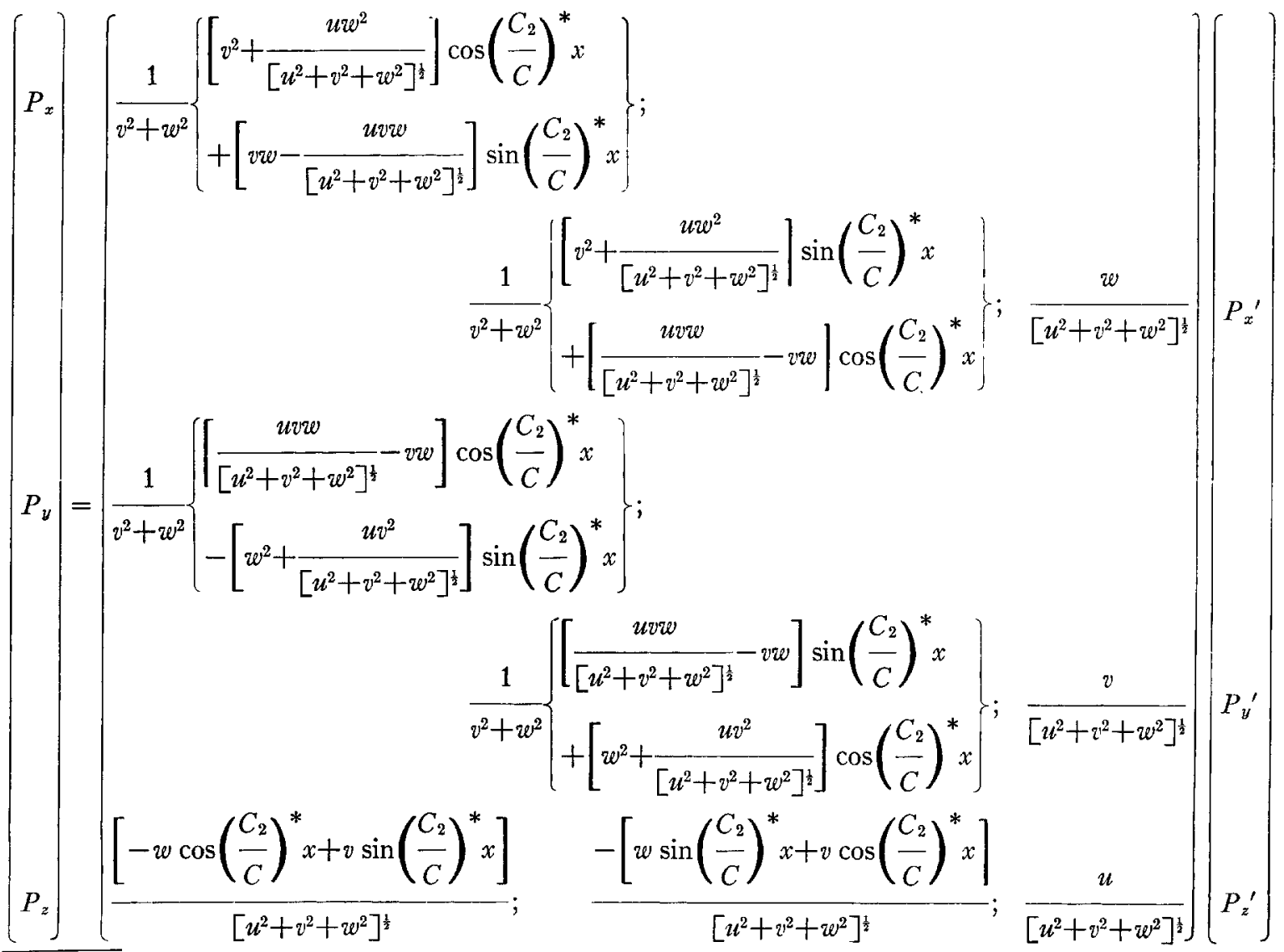

${ }^{13}$ N. Solimene and B. P. Dailey, J. Chem. Phys. 22, 2042 (1954).

${ }_{14}$ D. G. Burkhard, Trans. Faraday Soc. 52, 1 (1956). 
in which the following abbreviations have been used:

and where now

$$
u=A B-E^{2}, \quad v=A D+E F, \quad w=E D+B F,
$$

$$
\left(\frac{C_{2}}{C}\right)^{*}=\frac{C_{2}\left[u^{2}+\imath^{2}+w^{2}\right]^{\frac{1}{2}}}{A_{\text {principal }} \cdot B_{\text {principal }} \cdot C_{\text {principal }}} .
$$

The new form of the Hamiltonian is very similar to that of the simpler type of asymmetric hindered rotator and gives rise to the following matrix elements

$$
\begin{aligned}
& H_{J K \boldsymbol{r} n} J K r n=\frac{\hbar^{2}}{4}\left\{\frac{u(A+B)+v D+w F}{u^{2}+v^{2}+w^{2}}\right\}\left(J^{2}+J-K^{2}\right)+\frac{\hbar^{2}}{2}\left\{\frac{A v^{2}+B w^{2}+2 E v w}{u\left(u^{2}+v^{2}+w^{2}\right)}+\frac{u^{2}+v^{2}+w^{2}}{u(u C-v D-w F)}\right\} K^{2}+E_{K \tau n} \text { int } \\
& H_{J K \tau n}{ }^{J(K+1) \tau^{\prime} n^{\prime}}=\frac{\hbar^{2}}{2}(2 K+1)[(J-K)(J+K+1)]^{\frac{1}{2}} \int_{0}^{2 \pi} P_{K r n} *(x) e^{-i x} P_{(K+1) \tau^{\prime} n^{\prime}}(x) d x \\
& \cdot\left\{\left[\frac{v\left(A v^{2}+B w^{2}+2 E v w\right)}{\left(v^{2}+w^{2}\right)\left(u^{2}+v^{2}+w^{2}\right)}-\frac{w(w D-v F)}{\left(v^{2}+w^{2}\right)\left[u^{2}+v^{2}+w^{2}\right]^{\frac{1}{2}}}\right]\right. \\
& \left.+i\left\{\frac{w\left(A v^{2}+B w^{2}+2 E v w\right)}{\left(v^{2}+w^{2}\right)\left(u^{2}+v^{2}+w^{2}\right)}+\frac{v(w D-v F)}{\left(v^{2}+w^{2}\right)\left[u^{2}+v^{2}+w^{2}\right]^{\frac{1}{3}}}\right]\right\} \\
& H_{J K \tau n} J(K+2) \tau^{\prime \prime} n^{\prime}=-\frac{\hbar^{2}}{8}[(J-K)(J-K-1)(J+K+1)(J+K+2)]^{\frac{1}{2}} \int_{0}^{2 \pi} P_{K \tau n^{\prime}} *(x) e^{-2 i x} P_{(K+2) \tau^{\prime \prime} n^{\prime}}(x) d x \\
& \cdot\left\{\left[\frac{u(B-A)}{\left(u^{2}+v^{2}+w^{2}\right)}+\frac{\left(v^{4}-w^{4}\right)(v D+w F)-4 u^{2} v w(w D-v F)}{\left(u^{2}+v^{2}+w^{2}\right)\left(v^{2}+w^{2}\right)^{2}}+\frac{4 w w(w D-v F)}{\left(v^{2}+w^{2}\right)^{2}\left[u^{2}+v^{2}+w^{2}\right]^{1}}\right]\right. \\
& -i\left[\frac{u\left(v^{2}-w^{2}\right)(w D-v F)}{\left(v^{2}+w^{2}\right)^{2}\left[u^{2}+v^{2}+w^{2}\right]^{\frac{1}{2}}}+\frac{w w(v D+w F)}{\left(v^{2}+w^{2}\right)\left(u^{2}+v^{2}+w^{2}\right)}\right. \\
& \left.\left.+\frac{u v w\left(\left[A v^{2}+B w^{2}+2 E v w\right]-u[v D+w F]\right)}{\left(v^{2}+w^{2}\right)^{2}\left(u^{2}+v^{2}+w^{2}\right)}\right]\right\} .
\end{aligned}
$$

Except for the imaginary part of the coefficients of the off-diagonal elements, these matrix elements have exactly the same form as those for the simpler type of asymmetric hindered rotator. However, the internal energies, $E_{K r n}{ }^{\text {int }}$, must be computed with new modified values for $\sigma$ and $\left(C_{1} C_{2} / C\right)^{*}$

$$
\begin{gathered}
\sigma=-K\left\{1-\frac{C_{2}\left[u^{2}+v^{2}+w^{2}\right]^{\frac{1}{2}}}{A_{\text {principal }} \cdot B_{\text {principal }} \cdot C_{\text {principal }}}\right\} \\
=-K\left(\frac{C_{1}}{C}\right)^{*}, \\
\left(\frac{C_{2} C_{1}}{C}\right)^{*}=\frac{C_{2}\left[u C_{1}-v D-w F\right]}{A_{\text {principal }} \cdot B_{\text {principal }} \cdot C_{\text {principal }}} .
\end{gathered}
$$

The hindered rotation splittings will now be functions of the principal moments of inertia and five additional parameters, for example

$$
\Delta_{0},\left(\frac{C_{1}}{C}\right)^{*}, \quad u, \quad v, \quad w
$$

The situation becomes much more complicated for hindered rotators consisting of two asymmetric components..$^{15}$ For this type of molecule the moments of inertia of the entire molecule as well as the internal angular momentum of either component of the molecule, when viewed from a coordinate system fixed in the other component, will be functions of the internal rotation angle, $x$. For this reason the transformation to a molecule-fixed coordinate system can be accomplished, at least easily, only in zeroth order; and the matrix elements of the Hamiltonian will still contain complicated rotation-hindered rotation interaction terms.

${ }_{15}$ D. G. Burkhard, J. Chem. Phys. 21, 1541 (1953); D. G. Burkhard and J. C. Irvin, J. Chem. Phys. 23, 1405 (1955). 